
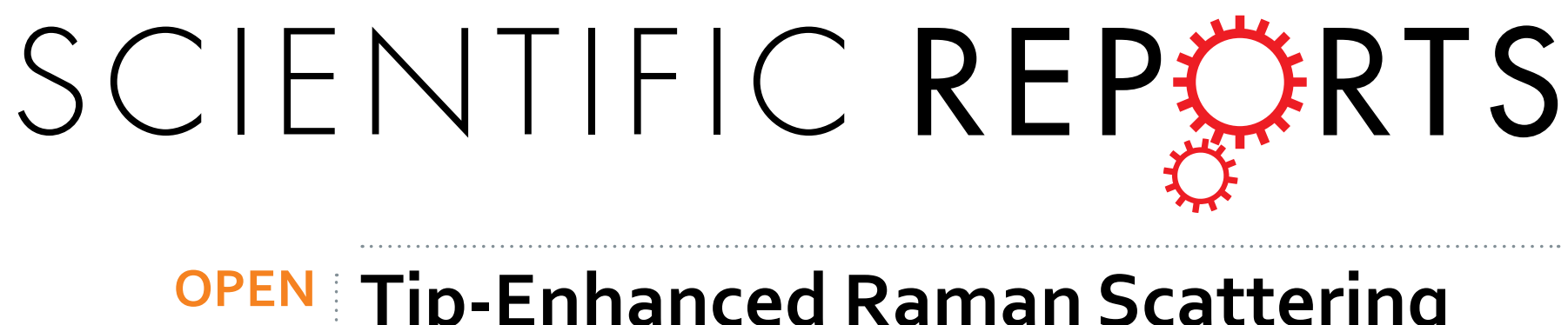

\title{
Tip-Enhanced Raman Scattering Imaging of Two-Dimensional Tungsten Disulfide with Optimized
}

Received: 13 September 2016

Accepted: 12 December 2016

Published: 13 January 2017

\section{Tip Fabrication Process}

\author{
Chanwoo Lee ${ }^{1}$, Sung Tae Kim ${ }^{1,2}$, Byeong Geun Jeong ${ }^{1}$, Seok Joon Yun ${ }^{1,2}$, Young Jae Song ${ }^{3,4}$, \\ Young Hee Lee ${ }^{1,2,4}$, Doo Jae Park ${ }^{5}$ \& Mun Seok Jeong ${ }^{1,2}$
}

We successfully achieve the tip-enhanced nano Raman scattering images of a tungsten disulfide monolayer with optimizing a fabrication method of gold nanotip by controlling the concentration of etchant in an electrochemical etching process. By applying a square-wave voltage supplied from an arbitrary waveform generator to a gold wire, which is immersed in a hydrochloric acid solution diluted with ethanol at various ratios, we find that both the conical angle and radius of curvature of the tip apex can be varied by changing the ratio of hydrochloric acid and ethanol. We also suggest a model to explain the origin of these variations in the tip shape. From the systematic study, we find an optimal condition for achieving the yield of $\sim 60 \%$ with the radius of $\sim 34 \mathrm{~nm}$ and the cone angle of $\sim 35^{\circ}$. Using representative tips fabricated under the optimal etching condition, we demonstrate the tip-enhanced Raman scattering experiment of tungsten disulfide monolayer grown by a chemical vapor deposition method with a spatial resolution of $\sim 40 \mathrm{~nm}$ and a Raman enhancement factor of $\sim 4,760$.

Fabrication of an ultrasharp metal nanotip is essential in various applications and state-of the-art instruments that are used in methods such as tip-enhanced Raman spectroscopy ${ }^{1,2}$, scattering-type near-field scanning optical microscopy $(\mathrm{s}-\mathrm{NSOM})^{3}$, scanning tunneling microscopy ${ }^{4,5}$, and ultrafast electron emission ${ }^{6-8}$. These ultrasharp metal nanotips enable high-resolution topographic imaging up to a few nanometers ${ }^{1,3,9-11}$ by virtue of the ultrasmall diameter (a few nanometers) of the tip apex. Additionally, the optical signal enhancement and spatial resolution, which are associated with the huge field enhancement and high field confinement, are also improved when such tips are used; this is particularly crucial when these nanotips are applied in instruments such as a tip-enhanced Raman spectroscope and in s-NSOM for chemical and energetic analysis of nanostructures such as nanowires ${ }^{12}$, carbon nanotubes ${ }^{1,11,13,14}$, graphene $\mathrm{e}^{15-18}$, and even single molecules ${ }^{9,11}$. Also, a control of cone angle of the nanotip is desirable to meet maximum Raman enhancement ${ }^{11}$.

Electrochemical etching is not only widely applied in the fabrication of ultrasharp metal nanotips but is also regarded as the most efficient method for fabricating a tip with an apex radius of a few tens of nanometers. Furthermore, electrochemical etching has advantages over other methods such as the pulling method ${ }^{19-23}$ in terms of a high yield, low cost, and short fabrication time. Other method reported by Johnson et. al. ${ }^{11}$ which have shown a fabrication of pyramidal tip with a capability of mass production and with a high reproducibility compared to the electrochemical etching. However, such tips are hardly applicable in top-illuminating optical configuration, which is necessary to an opaque sample. The electrochemical etching method involves the use of an electrolyte such as hydrochloric acid $(\mathrm{HCl})^{13,24}$. A direct current or an alternating current is applied to a metal wire, and electrically induced oxidation of metal via electron exchange with ions in solution etches the metal surface, ultimately forming a sharp edge. These processes are dominant in the meniscus of the solution around

${ }^{1}$ Department of Energy Science, Sungkyunkwan University (SKKU), Suwon 16419, Republic of Korea. ${ }^{2}$ Center for Integrated Nanostructure Physics, Institute for Basic Science (IBS), Suwon 16419, Republic of Korea. ${ }^{3}$ SKKU Advanced Institute of Nanotechnology (SAINT), Sungkyunkwan University, Suwon 16419, Republic of Korea. ${ }^{4}$ Department of Physics, Sungkyunkwan University, Suwon 16419, Republic of Korea. ${ }^{5}$ Department of Physics, Hallym University, Hallymdaehakgil 1, Chuncheon 24252, Republic of Korea. Correspondence and requests for materials should be addressed to D.J.P. (email: doojaepark@hallym.ac.kr) or M.S.J. (email: mjeong@skku.edu) 
(a)
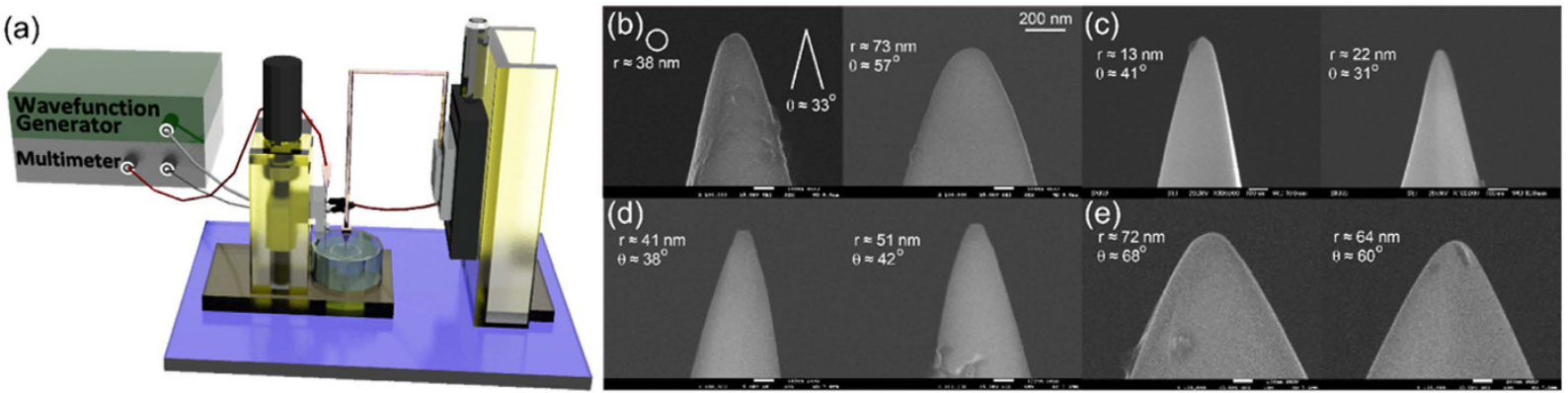

Figure 1. (a) Schematic diagram of the homemade electrochemical etching setup. A gold wire is connected to the positive pole (anode) of an arbitrary waveform generator to attract chloride ions and to initiate gold ionization. A platinum loop is connected to the negative pole (cathode) of the wave function generator. (b-e) SEM images of the etched gold tips fabricated with various concentrations of ethanol and $\mathrm{HCl}$ solution. (b) Only $\mathrm{HCl}$ solution. (c) $\mathrm{HCl}$ solution:ethanol = 3:1, (d) 2:1, and (e) 1:1 (Scale bar of right upper applies to all SEM images in $\mathbf{b}-\mathbf{e})$.

the metal wire owing to the high ion concentration, which is a key phenomenon for shaping the metal nanotip through electrochemical etching 25 .

However, when the chemical process is used, precise control of the ambient conditions (the temperature, humidity, etc.), applied bias, and ion concentration of the etching solution is required to fabricate a tip with the desired shape. Hence, a systematic study on the effect of the applied bias and ion concentration, which are related to the shape of the tip, should be performed. A series of studies has reported on the influence of the viscosity ${ }^{26}$ and the effect of the bias voltage ${ }^{27}$, including its amplitude ${ }^{25}$ and duty cycle ${ }^{20}$. Although a study about controlling the concentration of $\mathrm{HCl}$ solution using two different alcohols has been published ${ }^{24}$, it is essential to use only one control factor; this practice plays a key role in precisely controlling the concentration of ions in the etchant. In addition, a study on how the concentration of ions in the etchant determines the radius of curvature $\left(R_{\mathrm{c}}\right)$ of etched tips has not been conducted to date. Moreover, a number of previous reports of the electrochemical etching method to fabricate the gold nanotip for tip-enhanced Raman scattering (TERS) presented the reproducibility and estimated the applicability but did not present TERS images ${ }^{19-21,23,24,26,27}$. In this study, we performed a series of experiments to fabricate a gold nanotip by electrochemical etching using a $37 \% \mathrm{HCl}$ solution diluted with $99.9 \%$ anhydrous ethanol to examine the effect of the $\mathrm{HCl}$ concentration on the fabricated tip shape. From a statistical study of tips fabricated under various $\mathrm{HCl}$ concentrations, we found the optimal condition that produces a higher yield or sharper tip apex. Using electrochemically etched tips, we also obtained TERS images of an atomically thin tungsten disulfide $\left(\mathrm{WS}_{2}\right)$ monolayer grown by chemical vapor deposition (CVD).

\section{Results and Discussion}

Gold nanotip fabrication by using electrochemical etching method. The etched gold tips fabricated using $\mathrm{HCl}$ and ethanol solutions with various concentration ratios were observed using a scanning electron microscope (SEM) to examine the $R_{\mathrm{c}}$ value and cone angle of the tip apex. Figure la shows a schematic diagram of the electrochemical etching method (see the Methods section). SEM images of representative tips fabricated using four concentration ratios are shown in Fig. $1 \mathrm{~b}-\mathrm{e}(\mathrm{HCl}$ solution only and $\mathrm{HCl}$ :ethanol=3:1, 2:1, and 1:1). The tips fabricated in $\mathrm{HCl}$ alone were rather blunt, having $R_{\mathrm{c}}$ values of 38 and $73 \mathrm{~nm}$ and marginal cone angles of $33^{\circ}$ and $57^{\circ}$, respectively. At a lower $\mathrm{HCl}$ concentration (3:1 volume ratio), $R_{\mathrm{c}}$ decreases significantly to 13 and $22 \mathrm{~nm}$, and then increases slowly as the $\mathrm{HCl}$ concentration decreases further, as can be seen for the 2:1 and 1:1 volume ratios (parts $\mathrm{d}$ and e, respectively, in Fig. 1), where $R_{\mathrm{c}}$ is large $(72$ and $64 \mathrm{~nm}$ ) in the latter case. The cone angle of the tips does not seem to change significantly with decreasing $\mathrm{HCl}$ concentration except at the 1:1 volume ratio, which yielded large conical angles of $60^{\circ}$ and $68^{\circ}$. These figures show that the effect of the $\mathrm{HCl}$ concentration on the tip shape cannot be easily determined because other uncontrollable conditions such as the temperature fluctuation of the solution, humidity of the laboratory, and microscopic fluctuation of $\mathrm{HCl}$ concentration in the etchant may affect the electrochemical etching process.

To statistically rule out the effects of such uncontrollable conditions, we fabricated $\sim 100$ tips at a fixed volume ratio. To obtain meaningful statistical parameters, we first excluded those tips that showed clear failure of the etching process, having an irregularly shaped apex or an $R_{\mathrm{c}}$ value larger than $100 \mathrm{~nm}$ (Figure S2). On the other hand, the tips fabricated successfully regardless of the etching conditions can be used to perform the TERS experiment (Figure S3). Table 1 summarizes the success rate, average $R_{\mathrm{c}}$ value, and cone angle for different $\mathrm{HCl}$ concentrations. The success rate is $\sim 50 \%$ at the highest $\mathrm{HCl}$ concentration and increases gradually with decreasing $\mathrm{HCl}$ concentration, reaching $67 \%$ at a $2: 1$ volume ratio. However, the success rate drops suddenly at the lowest $\mathrm{HCl}$ concentration (1:1 volume ratio). This drop may be attributed to a reduced etching ratio, which potentially causes the gold wire immersed in the solution to suddenly fall away owing to gravitational forces, which may tear the gold wire in the etching region and ultimately introduce either an irregular apex shape or a blunt end. This scenario is also supported by the fact that the current drop was slower than that at other concentrations. Further, in Table 1, $R_{\mathrm{c}}$ decreases gradually as the $\mathrm{HCl}$ concentration decreases. At the highest concentration ( $\mathrm{HCl}$ only), the average $R_{\mathrm{c}}$ value was $53.7 \mathrm{~nm}$, and $R_{\mathrm{c}}$ shows a minimum of $21.7 \mathrm{~nm}$ at the lowest concentration. This trend can be attributed to a modification of the etching speed with variation in the $\mathrm{HCl}$ concentration, and corresponding variation 


\begin{tabular}{|l|c|c|c|c|c|}
\hline & \multirow{2}{*}{$\begin{array}{c}\text { Success } \\
\text { HCl: Ethanol Ratio }\end{array}$} & \multicolumn{2}{|c|}{ Radius of Curvature (nm) } & \multicolumn{2}{c|}{ Cone Angle $\left({ }^{\circ}\right)$} \\
\cline { 3 - 6 } & 49.1 & Average & Deviation & Average & Deviation \\
\hline HCl only & 58.7 & 34.4 & 29.1 & 54.9 & 16.7 \\
\hline 3: 1 & 66.7 & 43.0 & 21.4 & 35.2 & 10.4 \\
\hline 2: 1 & 21.7 & 21.2 & 12.1 & 68.5 & 29.2 \\
\hline 1: 1 & & & & & 26.4 \\
\hline
\end{tabular}

Table 1. Statistics for apex radius and cone angle of gold nanotips corresponding to the composition of $\mathrm{HCl}$ and ethanol solution (Criterion of success is tip having apex radius below $100 \mathrm{~nm}$ ).

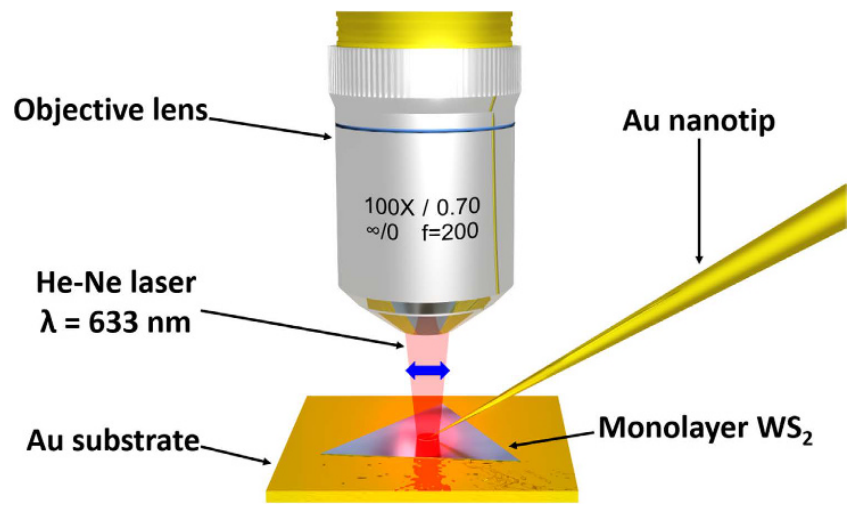

Figure 2. Schematic diagram of tip-enhanced Raman spectroscopy. Laser light having wavelength of $633 \mathrm{~nm}$ and output power of $\sim 1 \mathrm{~mW}$ is focused to the monolayer $\mathrm{WS}_{2}$ on a gold substrate by using an objective lens with a magnification of $100 \times$ and a numerical aperture of 0.7 . The blue arrow indicates a polarization direction of the laser.

in the shape of the meniscus formed at the upper surface of the solution close to the gold wire (Figure S5). At high concentrations, a deep dimple may be formed owing to the rapid etching speed (Figure S5). In this case, the area where active etching has occurred becomes narrower because the rise of the solution due to capillary force is restricted by the deep dimple formation, which introduces more accelerated etching rate at the neck of the dimple. When the diameter of the remaining gold wire becomes too small to support the mass of wire immersed in the solution, this region would be pulled by gravitational forces to introduce further narrowing, which acts positively to form a smaller apex. However, an accelerated etching speed may prevent this process, and simply cutting the wire will produce a relatively large $R_{\mathrm{c}}$. At low concentrations, however, a shallower dimple is formed because of the low etching rate, which causes the meniscus to rise higher, in contrast to that at a higher concentration. In this case, a relatively low etching rate can be maintained until the moment of apex formation. Hence, the pulling process may dominate, which consequently enables a smaller $R_{c}$. This scenario agrees well with the discussion of the sudden drop in the success rate at the lowest concentration: at a much reduced etching rate, pulling of the gold wire frequently breaks the neck of the dimple rather than forming a sharp apex.

These observations imply that there should be an optimal HCl concentration: gold wire tends to be cut when the concentration is too high and to fall away when it is too low. Our observation suggests that the volume ratio of $37 \% \mathrm{HCl}$ solution and $99.9 \%$ ethanol should be $3: 1$ for obtaining a sufficiently small $R_{\mathrm{c}}$ of $34 \mathrm{~nm}$ at a sufficiently high success rate of $59 \%$.

Tip-enhanced Raman scattering imaging. To test whether our tips can be successfully applied in TERS, we used fabricated a tip with small apex radius (Figure S6) and two-dimensional samples which indicate $\mathrm{WS}_{2}$ and graphene (Figure S7) in our TERS microscope, which was integrated with a scanning probe microscope, confocal microscope, and Raman scattering spectroscope (NTEGRA Spectra, NT-MDT). The excitation laser which is polarized along the tip-axis orientation (He-Ne laser, $\lambda=633 \mathrm{~nm}$ ) is focused on the sample by an objective lens with a numerical aperture of 0.7 and a magnification of $100 \times$, as shown in Fig. 2 . The Raman scattering intensity and spectra are recorded using a charge-coupled detector (Andor) cooled to $-70^{\circ} \mathrm{C}$ and equipped with a spectrometer containing an 1800 grooves $/ \mathrm{mm}$ grating blazed at $500 \mathrm{~nm}$. The test specimen was monolayer WS grown on $\mathrm{SiO}_{2} / \mathrm{Si}$ substrate by CVD. Monolayer $\mathrm{WS}_{2}$ shows strong photoluminescence (PL) emission from $\sim 1.93$ to $\sim 1.96 \mathrm{eV}$ because of a direct band gap ${ }^{28}$. When Raman scattering is measured using laser excitation at $633 \mathrm{~nm}$, the PL background can be observed in the Raman spectrum of monolayer $\mathrm{WS}_{2}{ }^{29}$. To avoid the PL background using charge transfer to the metal substrate, monolayer $\mathrm{WS}_{2}$ flakes were transferred onto a gold substrate, which was fabricated by coating a $\mathrm{SiO}_{2} / \mathrm{Si}$ substrate with gold $\sim 100 \mathrm{~nm}$ in thickness using thermal evaporation. Figure 3a shows an optical microscope image of monolayer $\mathrm{WS}_{2}$ flakes on the gold substrate, where bilayer $\mathrm{WS}_{2}$ grown arbitrarily during synthesis of monolayer $\mathrm{WS}_{2}$ is also visible. To determine the contrast in the Raman scattering signals and the Raman enhancement factor $(E F)$ of TERS, the spectra were recorded at the same position (bilayer position) of the sample in TERS (tip-on) and normal Raman scattering (tip-off) measurement, as 
(a)

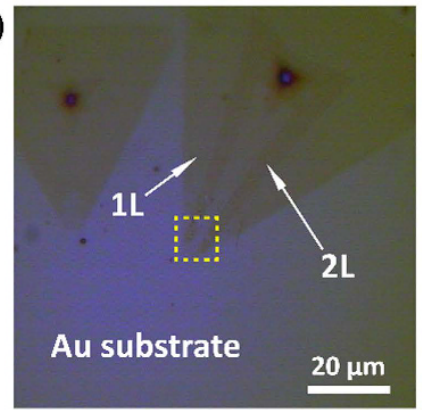

(b)

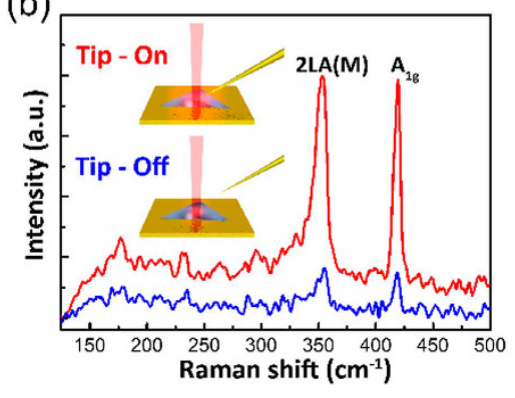

(c)

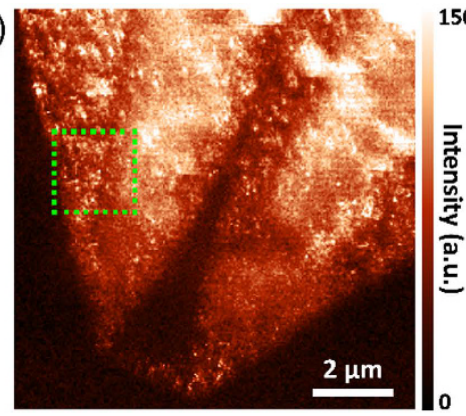

(d)

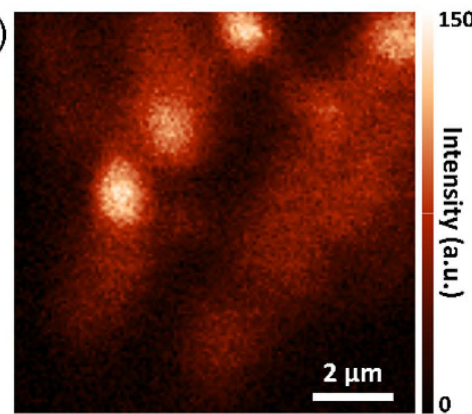

(e)

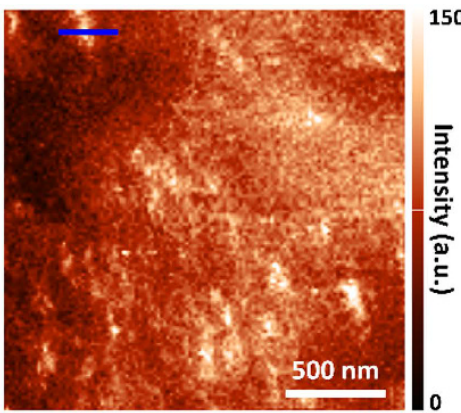

(f)

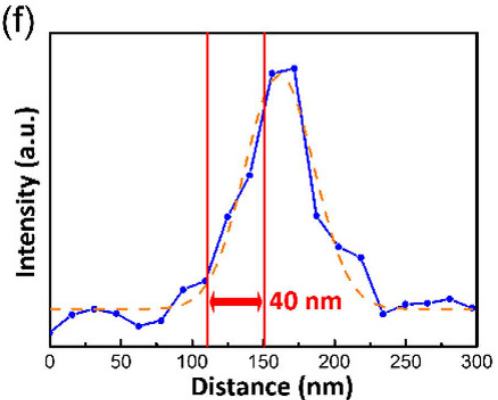

Figure 3. TERS spectra and images of mono/bilayer $\mathrm{WS}_{2}$ flake when applying a tip fabricated using a solution having 3:1 volume ratio of $\mathbf{H C l}$ and ethanol solutions. (a) Optical microscope image of the sample. (b) TERS spectrum (“Tip-on", red curve) and confocal Raman spectrum (“Tip-off”, blue curve) measured at the same position on the sample. (c) TERS image of $A_{1 g}$ peak area intensity (Yellow dashed box in (a) indicates TERS mapping area.). (d) Confocal Raman scattering image of $\mathrm{A}_{1 \mathrm{~g}}$ peak area intensity at the same position in (c). (e) Zoomed-in TERS image for a green dashed box in (c). (f) TERS intensity profile of the $\mathrm{A}_{1 \mathrm{~g}}$ peak signal along the blue bar in (e) and the fitted Gaussian peak (orange dashed line). The TERS spatial resolution by applying edge response function was obtained as $\sim 40 \mathrm{~nm}$.

shown in Fig. 3b. The intensities of the $\mathrm{A}_{1 \mathrm{~g}}$ peaks at $\sim 419 \mathrm{~cm}^{-1}$ were compared to determine the contrast and $E F$ of the TERS and confocal Raman scattering data. The contrast between the TERS and confocal Raman scattering intensities can be calculated using the amplitude ratio of the two peaks ( $I_{\text {Tip-on }}$ and $\left.I_{\text {Tip-off }}\right)$. The $E F$ value of TERS can also be calculated using the contrast, the radius of the laser spot $\left(R_{\text {Laser }}\right)$, and the $R_{\mathrm{c}}$ value of the tip $\left(R_{\text {tip }}\right)$. The equations for obtaining accurate contrast ${ }^{30}$ and $E F$ values in TERS $^{31}$ are defined as follows:

$$
\begin{gathered}
\text { Contrast }=\frac{I_{\text {Tip }-o n}-I_{\text {Tip }-o f f}}{I_{\text {Tip }-o f f}} \\
E F_{\text {TERS }}=\text { Contrast } \times\left(\frac{R_{\text {Laser }}}{R_{\text {Tip }}}\right)^{2}
\end{gathered}
$$

The value of the $\mathrm{A}_{1 \mathrm{~g}}$ peak contrast is calculated to be $\sim 5.2$. In addition, the radii of the excitation laser spot and the tip apex are $\sim 550$ and $\sim 18.2 \mathrm{~nm}$, respectively. Applying these values to eq (2), we obtain an $E F_{\text {TERS }}$ value of $\sim 4,760$. In addition, considering that the Raman scattering cross section is proportional to the fourth power of the electric field, we can also obtain an electric field enhancement factor of $\sim 8.3$, which is comparable to previously reported experimental results and theoretical predictions $s^{6,14}$. However, in practice, $E F_{\text {TERS }}$ value have to be smaller than the aforementioned value because of the enlargement of the tip field due to inclination of the tip ${ }^{32}$. Including this effect, actual $E F_{\text {TERS }}$ was obtained as $\sim 3,370$ when assuming tip inclination angle is $45^{\circ}$. Further, the analyses for a spatial resolution, which is related with TERS and confocal Raman scattering were carried out by showing a huge difference in the spatial resolution, as shown in Fig. 3c,d. Owing to the relatively weak Raman scattering signals and the diffraction-limited spatial resolution (Fig. 3d), it is difficult to distinguish the monolayer region and grain boundaries in the sample. In contrast to the confocal Raman scattering image in Fig. 3d, the TERS image in Fig. $3 \mathrm{c}$ shows the monolayer sample, boundaries between the monolayer and bilayer, and grain boundaries in monolayer $\mathrm{WS}_{2}$. To measure the spatial resolution, we acquired another TERS image with a step size of $15 \mathrm{~nm}$, as shown in Fig. 3e. The TERS intensity profile of the $\mathrm{A}_{1 \mathrm{~g}}$ peak along the blue bar in Fig. 3e represents the TERS spatial resolution, which reaches $\sim 40 \mathrm{~nm}$, as shown in Fig. $3 \mathrm{f}^{1,33}$. The high spatial resolution of TERS beyond the diffraction limit is $\sim 14$ times better than that of confocal Raman spectroscopy.

To confirm whether other tips fabricated under the optimal condition can be used to obtain TERS images, several tips etched under the 3:1 volume ratio condition were also used to conduct TERS imaging. Figure 4a shows an optical microscope image of a monolayer $\mathrm{WS}_{2}$ flake on the gold substrate as the tip approaches the sample. 
(a)

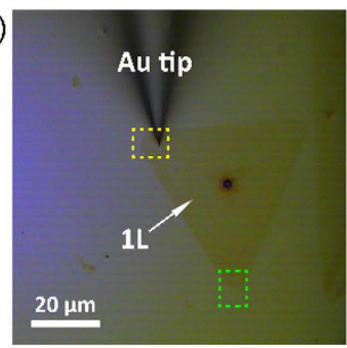

(c)

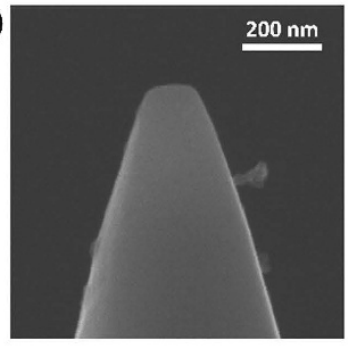

(e)

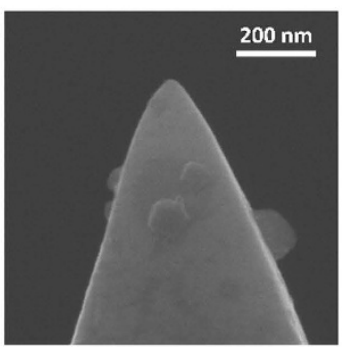

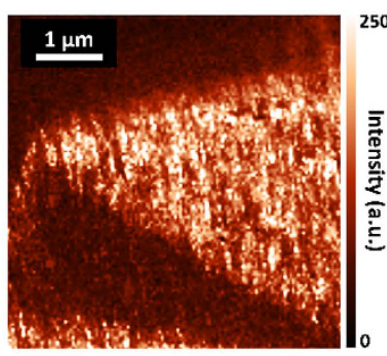

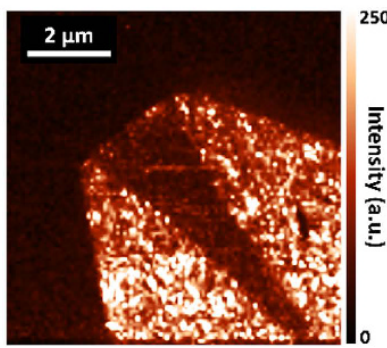

(b)
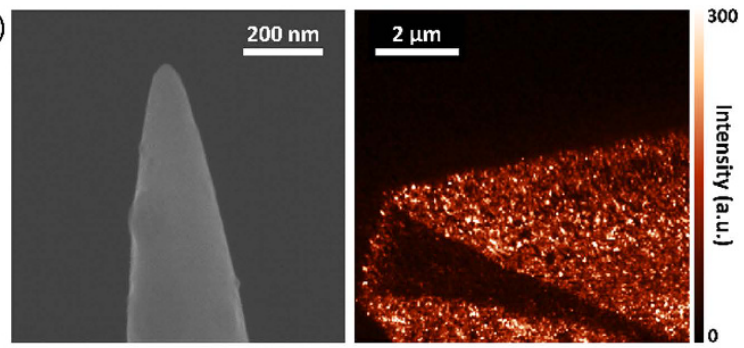

(d)
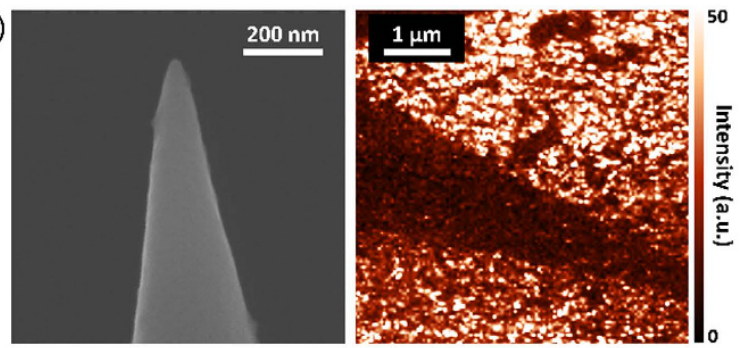

(f)
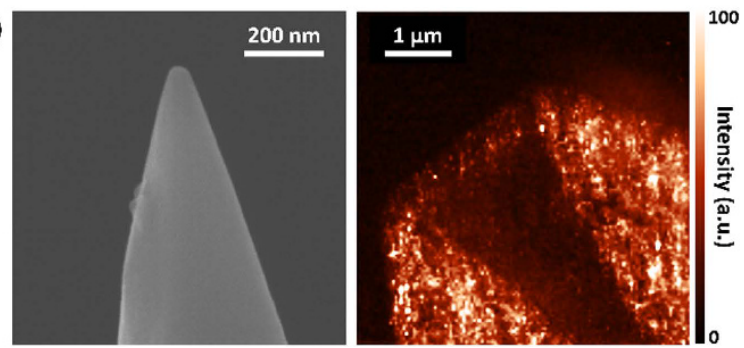

Figure 4. TERS images of $A_{1 \mathrm{~g}}$ peak area intensity of monolayer $\mathrm{WS}_{2}$ obtained by using several tips fabricated under the 3:1 volume ratio condition. (a) Optical microscope image of the tip approaching a sample. (b-d) Used tips and TERS images measured at a yellow dashed box in (a). (e, f) Used tips and TERS images measured at a green dashed box in (a).

Using different tips fabricated under the same condition, we successfully obtained various TERS images, as shown in Fig. 4b-f, even though radii and cone angles are slightly different each other. As shown in these figures, image qualities are different for various tip shape. For example, the image taken by rather blunt tip $(\sim 76 \mathrm{~nm})$ depicted in Fig. $3 \mathrm{c}$ shows much reduced resolution as comparing with the others. These results indicate that the tip fabricated under a 3:1 volume ratio of $\mathrm{HCl}$ solution and ethanol, which is suggested as an optimal condition, can be used to achieve greatly enhanced Raman scattering signals and high spatial resolution in Raman scattering images.

\section{Conclusions}

We determined the optimal composition of the $\mathrm{HCl}$ solution for fabrication of gold nanotips by electrochemical etching. We found that the optimum volume ratio of $\mathrm{HCl}$ and ethanol solution for obtaining a sufficiently small tip apex at an acceptable success rate was 3:1. We also demonstrated successful TERS spectroscopy for monolayer and bilayer $\mathrm{WS}_{2}$ flakes by using tips fabricated under this condition. We achieved a contrast of 5.2 and $E F_{\mathrm{TERS}}$ values as high as 4,760 when using an $18.2 \mathrm{~nm}$ radius of curvature. Further, we also obtained a TERS image with a high spatial resolution of $40 \mathrm{~nm}$, which is beyond the diffraction limit. We suggest that our method can contribute to high efficiency production of nanotips that are suitable for high efficiency TERS and also high resolution TERS imaging.

\section{Methods}

Electrochemical etching process. The gold nanotips were prepared using a homemade electrochemical etching system as shown in Fig. 1a. In this system, the gold wire (Nilaco Co.) to be etched is connected to a positive pole, and a platinum ring (Nilaco Co.) is connected to the negative pole of an arbitrary waveform generator, which supplies a series of pulsed electric biases with a peak amplitude of $3.5 \mathrm{~V}$, a background value of $-25 \mathrm{mV}$, a frequency of $300 \mathrm{~Hz}$, and a $20 \%$ duty cycle. This pulsed bias is expected to result in a relaxation time that allows the system to eliminate bubbles ${ }^{21}$ and any chemical instability caused by a violent reaction. The anode attracts anions such as chloride, which are generated near the cathode, to ionize the gold. As gold ionization progresses, the color of the $\mathrm{HCl}$ solution mixed with ethanol gradually changes from colorless and transparent to yellow because of precipitation of gold chloride (Figure S1). In our etching process, the platinum ring was first cleaned by immersing it in $36 \% \mathrm{HCl}$ solution (Duksan) for $5 \mathrm{~min}$, followed by rinsing with acetone, ethanol, deionized water, and isopropyl alcohol. To further remove any remaining chemicals, the ring was sonicated for $5 \mathrm{~min}$. This ring was immersed in $37 \% \mathrm{HCl}$ solution (Sigma-Aldrich) at an immersion depth of $\sim 2 \mathrm{~mm}$. A gold wire having 
a diameter of $250 \mu \mathrm{m}$ was submerged in the same solution at a depth of $\sim 0.5 \mathrm{~mm}$. To investigate the effect of the $\mathrm{HCl}$ concentration on the etching process, four solutions were prepared by mixing pure $37 \% \mathrm{HCl}$ solution and 99.9\% anhydrous ethanol (Duksan) in different volume ratios ( $\mathrm{HCl}$ only, 3:1, 2:1, and 1:1 for $\mathrm{HCl}$ : ethanol). An electrical bias with the properties described above was applied to the gold wire until the etching process was complete. A multimeter was used to monitor the current to assess the completion of the etching process. Such etching processes are usually self-terminating, and termination is manifested as a rapid decrease in current.

\section{References}

1. Chi Chen, N. H. S. K. A $1.7 \mathrm{~nm}$ resolution chemical analysis of carbon nanotubes by tip-enhanced Raman imaging in the ambient. Nat. Commun. 5, doi: 10.1038/ncomms4312 (2014).

2. Kumar, N., Stephanidis, B., Zenobi, R., Wain, A. J. \& Roy, D. Nanoscale mapping of catalytic activity using tip-enhanced Raman spectroscopy. Nanoscale 7, 7133-7137, doi: 10.1039/c4nr07441f (2015).

3. Schmidt, S. et al. Adiabatic nanofocusing on ultrasmooth single-crystalline gold tapers creates a 10-nm-sized light source with fewcycle time resolution. ACS Nano 6, 6040-6048, doi: 10.1021/nn301121h (2012).

4. Xue, J. et al. Scanning tunnelling microscopy and spectroscopy of ultra-flat graphene on hexagonal boron nitride. Nat Mater 10, 282-285, doi: http://www.nature.com/nmat/journal/v10/n4/abs/nmat2968.html\#supplementary-information (2011).

5. Tapaszto, L. et al. Breakdown of continuum mechanics for nanometre-wavelength rippling of graphene. Nat Phys 8, 739-742, doi: http://www.nature.com/nphys/journal/v8/n10/abs/nphys2389.html\#supplementary-information (2012).

6. Park, D. J. et al. Strong field acceleration and steering of ultrafast electron pulses from a sharp metallic nanotip. Phys Rev Lett 109, doi: 10.1103/PhysRevLett.109.244803 (2012).

7. Ropers, C., Solli, D. R., Schulz, C. P., Lienau, C. \& Elsaesser, T. Localized multiphoton emission of femtosecond electron pulses from metal nanotips. Phys Rev Lett 98, doi: 10.1103/PhysRevLett.98.043907 (2007).

8. Schenk, M., Krüger, M. \& Hommelhoff, P. Strong-field above-threshold photoemission from sharp metal tips. Phys Rev Lett 105, doi: 10.1103/PhysRevLett.105.257601 (2010).

9. Zhang, R. et al. Chemical mapping of a single molecule by plasmon-enhanced Raman scattering. Nature 498, 82-86, doi: 10.1038/ nature12151 http://www.nature.com/nature/journal/v498/n7452/abs/nature12151.html\#supplementary-information (2013).

10. Rang, M. et al. Optical Near-Field Mapping of Plasmonic Nanoprisms. Nano Lett. 8, 3357-3363, doi: 10.1021/nl801808b (2008).

11. Johnson, T. W. et al. Highly Reproducible Near-Field Optical Imaging with Sub-20-nm Resolution Based on Template-Stripped Gold Pyramids. ACS Nano 6, 9168-9174, doi: 10.1021/nn303496g (2012).

12. Böhmler, M., Wang, Z., Myalitsin, A., Mews, A. \& Hartschuh, A. Optical Imaging of CdSe Nanowires with Nanoscale Resolution. Angewandte Chemie International Edition 50, 11536-11538, doi: 10.1002/anie.201105217 (2011).

13. Park, K. D. et al. Laser fabrication of gold nanoparticle clustered tips for use in apertureless near-field scanning optical microscopy. J. Nanosci. Nanotechnol. 14, 5961-5964, doi: 10.1166/jnn.2014.8294 (2014).

14. Cançado, L. G., Hartschuh, A. \& Novotny, L. Tip-enhanced Raman spectroscopy of carbon nanotubes. J. Raman Spectrosc. 40, 1420-1426, doi: 10.1002/jrs.2448 (2009).

15. Wang, P. et al. Reversible Defect in Graphene Investigated by Tip-Enhanced Raman Spectroscopy. Proc SPIE Int Soc Opt Eng 7, 555-561, doi: 10.1007/s11468-012-9342-8 (2012).

16. Vantasin, S. et al. Tip-Enhanced Raman Scattering of the Local Nanostructure of Epitaxial Graphene Grown on $4 \mathrm{H}-\mathrm{SiC}\left(0001^{-}\right)$. The Journal of Physical Chemistry C 118, 25809-25815, doi: 10.1021/jp508730y (2014).

17. Farshid Pashaee, F. S. Giovanni Fanchini and François Lagugné-Labarthet. Tip-enhanced Raman spectroscopy of graphene-like and graphitic platelets on ultraflat gold nanoplates. Phys. Chem. Chem. Phys. 17, 21315-21322, doi: 10.1039/C4CP05252H (2015).

18. Beams, R., Cançado, L. G., Oh, S.-H., Jorio, A. \& Novotny, L. Spatial Coherence in Near-Field Raman Scattering. Phys Rev Lett 113, 186101 (2014).

19. Kim, P. et al. Efficient electrochemical etching method to fabricate sharp metallic tips for scanning probe microscopes. Rev. Sci. Instrum. 77, 103706, doi: http://dx.doi.org/10.1063/1.2358703 (2006).

20. Kharintsev, S. S., Hoffmann, G. G., Fishman, A. I. \& Salakhov, M. K. Plasmonic optical antenna design for performing tip-enhanced Raman spectroscopy and microscopy. J Phys D 46, 145501 (2013).

21. Ren, B., Picardi, G. \& Pettinger, B. Preparation of gold tips suitable for tip-enhanced Raman spectroscopy and light emission by electrochemical etching. Rev. Sci. Instrum. 75, 837-841, doi: http://dx.doi.org/10.1063/1.1688442 (2004).

22. Libioulle, L., Houbion, Y. \& Gilles, J. M. Very sharp gold and platinum tips to modify gold surfaces in scanning tunneling microscopy. Journal of Vacuum Science \&amp; Technology B 13, 1325-1331, doi: http://dx.doi.org/10.1116/1.587847 (1995).

23. Billot, L., Berguiga, L., de la Chapelle, M. L., Gilbert, Y. \& Bachelot, R. Production of gold tips for tip-enhanced near-field optical microscopy and spectroscopy: analysis of the etching parameters. The European Physical Journal-Applied Physics 31, 139-145, doi: 10.1051/epjap:2005049 (2005).

24. Kharintsev, S. S., Rogov, A. M. \& Kazarian, S. G. Nanopatterning and tuning of optical taper antenna apex for tip-enhanced Raman scattering performance. Rev. Sci. Instrum. 84, 093106, doi: http://dx.doi.org/10.1063/1.4822274 (2013).

25. Williams, C. \& Roy, D. Fabrication of gold tips suitable for tip-enhanced Raman spectroscopy. J Vac Sci Technol B Microelectron Nanometer Struct 26, 1761-1764, doi: 10.1116/1.2981078 (2008).

26. Park, J., Hong, T. S., Lee, N. S., Kim, K. B. \& Seo, Y. Viscosity dependence of electrochemical etching for gold tip fabrication. Curr. Appl. Phys. 11, 1332-1336, doi: http://dx.doi.org/10.1016/j.cap.2011.03.075 (2011).

27. Li, M. et al. Electrochemical fabrication of silver tips for tip-enhanced Raman spectroscopy assisted by a machine vision system. J. Raman Spectrosc. 47, 808-812, doi: 10.1002/jrs.4898 (2016).

28. Kim, M. S. et al. Biexciton Emission from Edges and Grain Boundaries of Triangular WS2 Monolayers. ACS Nano 10, 2399-2405, doi: 10.1021/acsnano.5b07214 (2016).

29. Zhao, W. et al. Lattice dynamics in mono- and few-layer sheets of WS2 and WSe2. Nanoscale 5, 9677-9683, doi: 10.1039/ C3NR03052K (2013).

30. Stadler, J., Schmid, T. \& Zenobi, R. Developments in and practical guidelines for tip-enhanced Raman spectroscopy. Nanoscale 4, 1856-1870, doi: 10.1039/C1NR11143D (2012).

31. Kumar, N., Rae, A. \& Roy, D. Accurate measurement of enhancement factor in tip-enhanced Raman spectroscopy through elimination of far-field artefacts. Applied Physics Letters 104, doi: 10.1063/1.4869184 (2014).

32. Pettinger, B., Schambach, P., Villagómez, C. J. \& Scott, N. Tip-Enhanced Raman Spectroscopy: Near-Fields Acting on a Few Molecules. Annual Review of Physical Chemistry 63, 379-399, doi: 10.1146/annurev-physchem-032511-143807 (2012).

33. Liao, M. et al. Tip-Enhanced Raman Spectroscopic Imaging of Individual Carbon Nanotubes with Subnanometer Resolution. Nano Lett. 16, 4040-4046, doi: 10.1021/acs.nanolett.6b00533 (2016).

\section{Acknowledgements}

This work was supported by IBS-R011-D1 and the Human Resources Development program (No. 20124010203270) of the Korea Institute of Energy Technology Evaluation and Planning (KETEP) grant funded by the Korean 
government Ministry of Trade, Industry and Energy. This research was also supported by the Hallym University Research Fund, 2016[HRF-201609-006]. We are also grateful to Yongmin Kim, a faculty of the Department of Applied Physics, Dankook University, for insightful discussions.

\section{Author Contributions}

C.L. and S.T.K. designed the experiment system, which fabricates gold nanotips by using electrochemical etching method. C.L. and B.G.J. fabricated the gold nanotips used to measure TERS images. C.L. performed TERS experiment. S.J.Y. performed synthesis experiments for tungsten disulfide nanosheets and Y.J.S. performed growth experiments for graphene. D.J.P. conducted the simulation for gold nanotips. C.L., Y.H.L, D.J.P., and M.S.J. contributed to experiment planning, data analysis, and manuscript preparation.

\section{Additional Information}

Supplementary information accompanies this paper at http://www.nature.com/srep

Competing financial interests: The authors declare no competing financial interests.

How to cite this article: Lee, C. et al. Tip-Enhanced Raman Scattering Imaging of Two-Dimensional Tungsten Disulfide with Optimized Tip Fabrication Process. Sci. Rep. 7, 40810; doi: 10.1038/srep40810 (2017).

Publisher's note: Springer Nature remains neutral with regard to jurisdictional claims in published maps and institutional affiliations.

(c) (i) This work is licensed under a Creative Commons Attribution 4.0 International License. The images or other third party material in this article are included in the article's Creative Commons license, unless indicated otherwise in the credit line; if the material is not included under the Creative Commons license, users will need to obtain permission from the license holder to reproduce the material. To view a copy of this license, visit http://creativecommons.org/licenses/by/4.0/

(C) The Author(s) 2017 